\title{
From ant trails to pedestrian dynamics
}

\author{
Andreas Schadschneider,' Ansgar Kirchner,' Katsuhiro Nishinari ${ }^{2}$ \\ 'Institute for Theoretical Physics, Universität zu Köln, Köln, Germany; ${ }^{2}$ Department of Applied Mathematics and \\ Informatics, Ryukoku University, Shiga, Japan
}

\begin{abstract}
This paper presents a model for the simulation of pedestrian dynamics inspired by the behaviour of ants in ant trails. Ants communicate by producing a pheromone that can be smelled by other ants. In this model, pedestrians produce a virtual pheromone that influences the motion of others. In this way all interactions are strictly local, and so even large crowds can be simulated very efficiently. Nevertheless, the model is able to reproduce the collective effects observed empirically, eg the formation of lanes in counterflow. As an application, we reproduce a surprising result found in experiments of evacuation from an aircraft.
\end{abstract}

Keywords: collective phenomena, traffic flow, computer simulations, evacuation processes, cellular automata, chemotaxis

\section{Introduction}

During the last decade, considerable research has been devoted to the topic of highway traffic using methods from physics (Chowdhury et al 2000; Helbing 2001). However, pedestrian dynamics (Schreckenberg and Sharma 2001) has not been studied as extensively as vehicular traffic. One reason is probably its generically two-dimensional nature. Therefore, only few models exist that can reproduce the empirically observed behaviour accurately. An important example is the 'social force model' (Helbing and Molnar 1995; Helbing 2001), where pedestrians are treated as particles ${ }^{1}$ subject to long-ranged forces induced by the social behaviour of the individuals. This leads to (coupled) equations of motion similar to Newtonian mechanics. Such a description has many similarities with the modelling of sand and other granular materials.

Due to the complexity, any pedestrian model for realistic applications has to be treated on a computer. Efficient computer simulations of the social force model are difficult for large crowds consisting of hundreds or thousands of individuals. Due to the long-ranged nature of forces, many interaction terms have to be calculated. An additional difficulty arises in complex geometries. It has to be checked whether an interaction between two individuals is possible, or whether it is blocked by walls. This requires complicated algorithms, which slow down the speed of the simulations.

Efficient computer simulations require simple models that nevertheless provide an accurate description of reality. One simple class of models are so-called cellular automata that have been studied in statistical physics for a long time (Wolfram 1986, 2002). In a cellular automaton, space, time and state variables are discrete, which makes them ideally suited for high-performance computer simulations. However, this still leaves the problem of the long-ranged interactions. In principle, a model is needed where the interactions are only local (restricted to the nearest neighbourhood) so that the presence of thousands of other people and walls does not influence performance of the simulations. It is exactly at this point, where we can learn from nature. The problem of transforming the effects of a long-ranged spatial interaction into local ones has already been solved by many insects. In fact, we will develop a model for pedestrian dynamics that is inspired by the formation of ant trails (Wilson 1971; Hölldobler and Wilson 1990; Camazine et al 2001; Mikhailov and Calenbuhr 2002). Here, the phenomenon of 'chemotaxis' is the clue (see BenJacob (1997) for a review). The model, which is introduced in the section Modelling pedestrian dynamics, uses a form of 'virtual chemotaxis' to mediate the interactions between the pedestrians.

To achieve complex behaviour in a simple fashion, one often resorts to a stochastic description. A realistic situation can seldom be described completely by a deterministic approach. Minor events can lead to a very different behaviour due to the complexity of the interactions involved. For the problem of pedestrian motion this becomes evident in the case of a panic, for example, where the behaviour of people seems almost unpredictable. But also for 'normal' situations, a stochastic component in the dynamics can lead to a more accurate description of complex phenomena, since

\footnotetext{
Correspondence: Andreas Schadschneider, Institute for Theoretical Physics, Universität zu Köln, Köln 50937, Germany; tel +49 221 470 43 12; fax +49 22I 470 5159; email as@thp.uni-koeln.de
} 
a

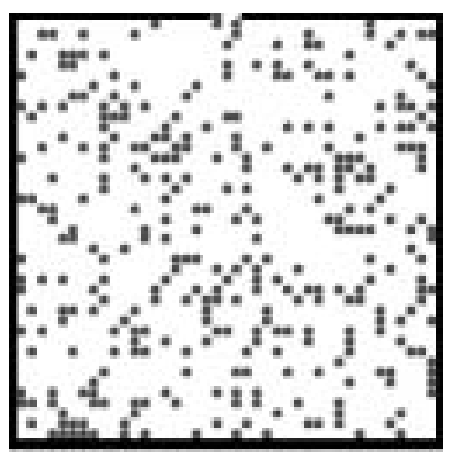

b

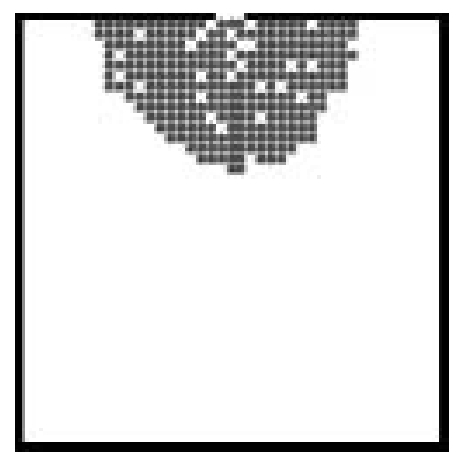

c

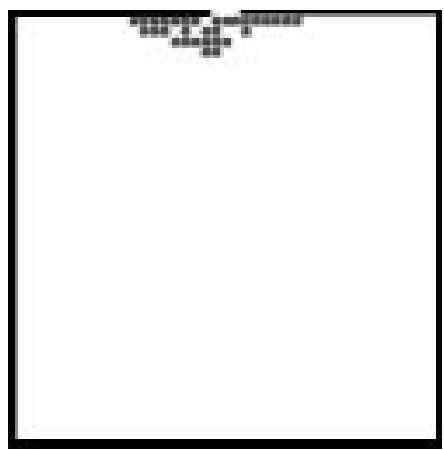

Figure I Clogging near a bottleneck (door). Shown are typical stages of an evacuation simulation: initial state $(t=0)(a)$; middle stages $(b)$; end stage with only a few particles left (c).

it takes into account that we usually do not have full knowledge about the state of the system and its dynamics.

\section{Collective phenomena}

One of the reasons why the investigation of pedestrian dynamics is attractive for physicists is that many interesting collective effects and self-organisation phenomena can be observed. Here, we give only a brief overview; refer to Helbing (2001) and Schreckenberg and Sharma (2001) for a more comprehensive discussion and list of references.

\section{Jamming}

At large densities, various kinds of jamming phenomena occur; for example, when many people try to leave a large room at the same time, and the flow is limited by a door or narrowing (see Figure 1). Therefore, this kind of jamming phenomenon does not depend strongly on the microscopic dynamics of the particles. This clogging effect is typical for a bottleneck situation. It is important for practical applications, especially evacuation simulations.

Other types of jamming occur in the case of counterflow where two groups of pedestrians mutually block each other. This happens typically at high densities and when it is not

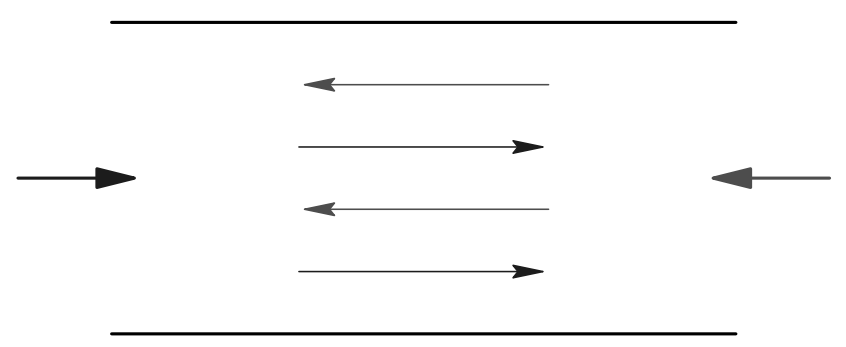

Figure 2 Lane formation in counterflow in a narrow corridor. possible to turn around and move back, eg when the flow of people is large.

\section{Lane formation}

In counterflow, ie two groups of people moving in opposite directions, a kind of spontaneous symmetry breaking occurs (see Figure 2). The motion of the pedestrians can selforganise in such a way that (dynamically varying) lanes are formed where people move in just one direction (Helbing and Molnar 1995). In this way, strong interactions with oncoming pedestrians are reduced, and a higher walking speed is possible.

\section{Oscillations}

In counterflow at bottlenecks, eg doors, one can observe oscillatory changes in the direction of motion. Once a pedestrian is able to pass the bottleneck, it becomes easier for others to follow her/him in the same direction until somebody is able to pass the bottleneck (eg through a fluctuation) in the opposite direction (see Figure 3).

\section{Patterns at intersections}

At intersections, various collective patterns of motion can be formed. Typical examples are short-lived roundabouts, which make the motion more efficient. Even if these are connected with small detours, the formation of these patterns can be favourable since they allow for a 'smoother' motion.

\section{Panic situations}

In panic situations, many counter-intuitive phenomena can occur. In the 'faster-is-slower effect', a higher desired velocity leads to a slower movement of a large crowd 


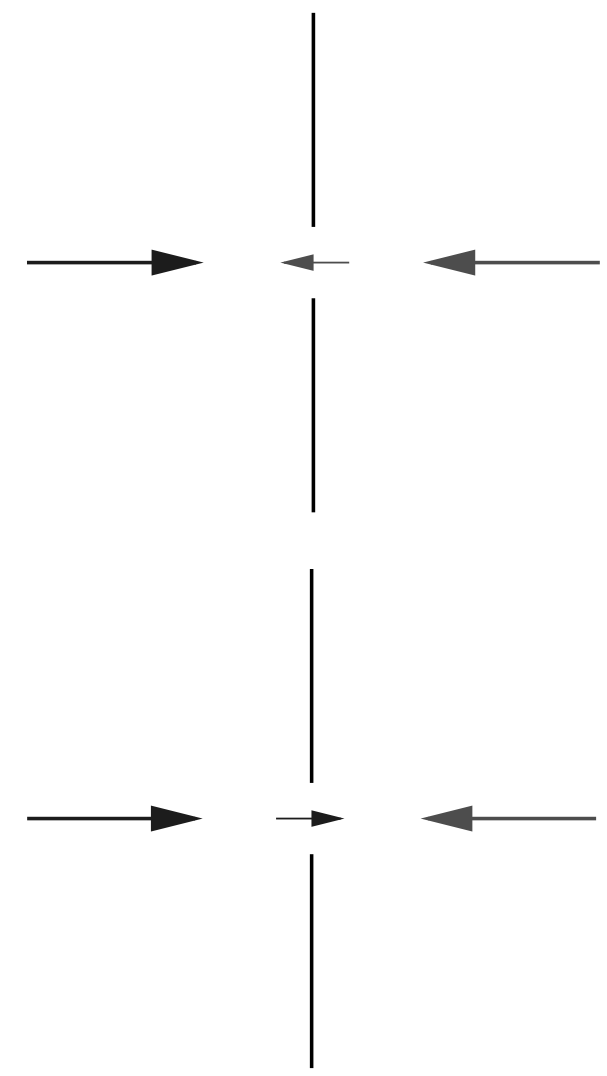

Figure 3 Oscillations of the flow direction at a door with counterflow.

(Helbing et al 2000). Such effects are extremely important for evacuations in emergency situations.

\section{Modelling ant trails}

Before introducing our model of pedestrian dynamics, we will discuss a model for ant trails (Chowdhury et al 2002; Nishinari et al 2003), which shows the basic principles, especially the influence of chemotaxis.

Ants communicate with each other by dropping a chemical (generically called 'pheromone') on the substrate as they crawl forward (Wilson 1971; Hölldobler and Wilson 1990; Camazine et al 2001; Mikhailov and Calenbuhr 2002). Although we cannot smell it, the trail pheromone sticks to the substrate long enough for the other following ants to pick up its smell and follow the trail. Ant trails may serve different purposes (trunk trails, migratory routes) and may also be used in a different way by different species. Therefore, one-way trails are observed as well as trails with counterflow of ants.

Here, we consider one-dimensional trails with ants moving only in one direction. The trail is now divided into cells that can accommodate at most one ant at a time (see
Figure 4). The cells are labelled by the index $i(i=1,2, \ldots, L)$; $L$ being the length of the lattice. With each ant cell, we associate another cell for the pheromones (Figure 4). For simplicity, only the two states 'pheromone present' and 'no pheromone present' are noted.

The dynamics of the model consists of two stages (see Figure 4). In stage I, ants are allowed to move, whereas stage II corresponds to the dynamics of the pheromone.

\section{Stage I: motion of ants}

An ant in cell $i$ that has an empty cell in front of it moves forward with:

$$
\text { probability }=\begin{array}{cl}
-Q & \text { if a pheronome is present at } i+1 \\
-\mathcal{Q} & \text { if no pheromone is present at } i+1
\end{array}
$$

where, to be consistent with real ant trails, we assume $q<Q$. The value of the hopping probability represents the average velocity $\bar{v}$ of non-interacting ants. Without pheromones, a single ant moves with $\bar{v}=q$, whereas $\bar{v}=Q$ in the presence of pheromones. Thus, stage I, in a simple way, represents the fact that ants move faster towards their destination if they are guided by pheromones.

\section{Stage II: evaporation of pheromones}

At each cell $i$ occupied by an ant after stage I, a pheromone will be created. In this way, a kind of 'pheromone trace' is produced by moving ants. Any 'free' pheromone at a site $i$ not occupied by an ant will evaporate with the probability $f$

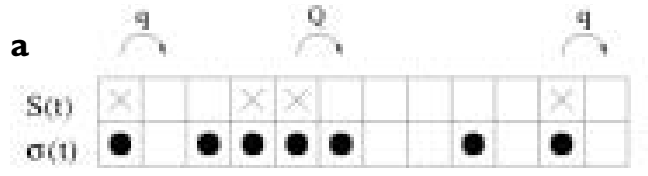

ants pleromone

b

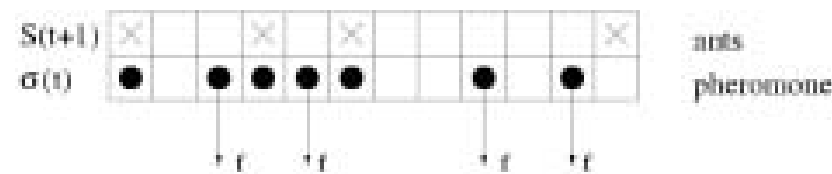
c

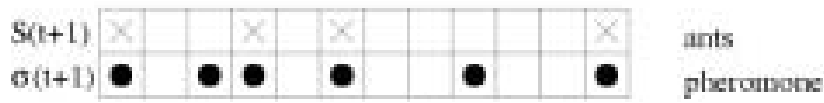

Figure 4 Schematic representation of typical configurations; it also illustrates the update procedure. (a) Configuration at time $t$, ie before stage I of the update. The non-vanishing hopping probabilities of the ants are also shown explicitly. (b) Configuration after one possible realisation of stage I. Two ants have moved compared with the top part of the figure. Also indicated are the pheromones that may evaporate in stage II of the update scheme. (c) Configuration after one possible realisation of stage II. Two pheromones have evaporated and one pheromone has been created due to the motion of an ant. 
per unit time; therefore, the trace created by a moving ant will disappear after some time if it is not renewed by other ants.

Despite its simplicity, this model contains the basics that describe the motion of ants in a trail (Chowdhury et al 2002; Nishinari et al 2003). The forward motion is represented by hopping, whereas the mutual hindrance of ants comes from the exclusion principle that allows at most one ant per cell. The influence of the pheromone is encoded in the hopping probabilities $q$ and $Q$, and finally it has also been taken into account that pheromones have a finite lifetime through the evaporation probability $f$. Of course, many subtle details have been neglected. However, these details turn out to be of less importance for an understanding of the basic principles underlying the process of chemotaxis.

\section{Modelling pedestrian dynamics}

In the previous section, the basic principles for modelling ant trails was presented. Based on similar principles, a model for pedestrian dynamics was developed (Burstedde et al 2001; Kirchner and Schadschneider 2002). Guided by the phenomenon of chemotaxis the interactions between pedestrians are local and thus allow for computational efficiency.

\section{General principles}

The pedestrians create a 'virtual trace', which then influences the motion of other pedestrians. This allows for a very efficient implementation on a computer since now all interactions are local. The transition probabilities for all pedestrians only depend on the occupation numbers and strength of the virtual trace in the neighbourhood, ie the long-ranged spatial interaction has been translated into a local interaction with 'memory'. The number of interaction terms in other long-ranged models, eg the social-force model, is of the order $N^{2}$ (where $N$ is the number of particles), whereas it is only of the order $N$ in our model.

The idea of a virtual trace can be generalised to a socalled 'floor field'. This floor field includes the virtual trace created by the pedestrians as well as a static component, which does not change with time. The latter allows modelling, eg preferred areas, walls and other obstacles. The pedestrians then react to both types of floor fields.

To keep the model simple, the particles needed to be provided with as little 'intelligence' as possible, and there needed to be the formation of complex structures and collective effects by means of self-organisation. In contrast to other approaches, it is not necessary to make detailed assumptions about human behaviour. Nevertheless, the model is able to reproduce many of the basic phenomena.

The key feature to substitute individual intelligence is the floor field. Apart from the occupation number, each cell carries an additional quantity (field) similar to the pheromone field in the ant trail model discussed. This field has its own dynamics given by diffusion and decay.

Interactions between pedestrians are repulsive for short distances. One likes to keep a minimal distance from others to avoid bumping into them. In the simplest version of the model, this is taken into account through hard-core repulsion, which prevents multiple occupation of the cells. For longer distances, the interaction is often attractive; for example, when walking in a crowded area it is usually advantageous to follow directly behind the predecessor. Large crowds may also be attractive due to curiosity.

These basic principles are already sufficient to reproduce the effects described in the section Collective phenomena, which so far only has been achieved by the social force model (Helbing and Molnar 1995). This shows the ability of cellular automata to create complex behaviour out of simple rules, and the great applicability of this approach to all kinds of traffic flow problems (Chowdhury et al 2000).

\section{Definition of the model and its dynamics}

The area available for pedestrians is divided into small cells of approximately $40 \mathrm{~cm} \times 40 \mathrm{~cm}$. This is the typical space occupied by a pedestrian in a dense crowd. As in the ant trail model, each cell can either be empty or occupied by exactly one particle (pedestrian). For special situations it might be desirable to use a finer discretisation, such that each pedestrian occupies four cells, for example, instead of one. Each pedestrian can move to one of the unoccupied next-neighbour cells $(i, j)$ (or stay at the present cell) at each discrete time step $t \rightarrow t+1$, according to certain transition probabilities $p_{i j}$ (Figure 5) as explained below.
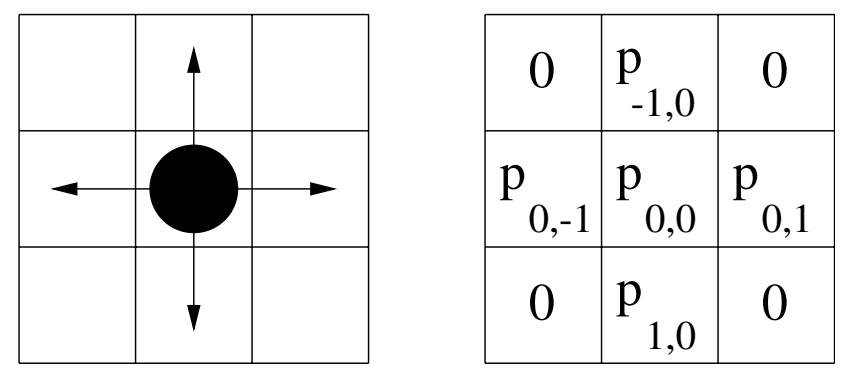

Figure 5 Possible target cells for a person at the next time step. 
For the case of evacuation processes, the 'static floor field' $S$ describes the shortest distance to an exit door. The field strength $S_{i j}$ is set inversely proportional to the distance from the door. The 'dynamic floor field' $D$ is a 'virtual trace' left by the pedestrians similar to the pheromone in chemotaxis (see Ben-Jacob (1997) for a review). It has its own dynamics, namely diffusion and decay, which leads to broadening, dilution and finally vanishing of the trace. At $t=0$ for all sites $(i, j)$ of the lattice, the dynamic field is zero, ie $D_{i j}=0$. Whenever a particle jumps from site $(i, j)$ to one of the neighbouring cells, $D$ at the origin cell is increased by one.

The update is done in parallel for all particles (synchronous dynamics). This introduces a time scale into the dynamics, which can roughly be identified with the reaction time $t_{\text {reac }}$. From the empirically observed average velocity of a pedestrian of about $1.3 \mathrm{~m} / \mathrm{s}$, one can estimate that one time step in our model corresponds to approximately $0.3 \mathrm{~s}$ in real time. This is of the order of the reaction time $t_{\text {reac }}$ and thus consistent with our microscopic rules. It also agrees nicely with the time needed to reach the normal walking speed, which is about $0.5 \mathrm{~s}$.

The update rules of our cellular automaton are divided into five steps that have the following structure:

1. The dynamic floor field $D$ is modified according to its diffusion and decay rules, controlled by the parameters $\alpha$ and $\delta$. In each time step of the simulation, each single particle of the whole dynamic field $D$ decays with probability $\delta$ and diffuses with probability $\alpha$ to one of its neighbouring cells.

2. For each pedestrian, the transition probabilities $p_{i j}$ for a move to an unoccupied next-neighbour cell $(i, j)$ are determined by the two floor fields and one's inertia (Figure 5). The values of the fields $D$ (dynamic) and $S$ (static) are weighted with two sensitivity parameters $k_{D}$ and $k_{S}$ :

$$
p_{i j}=N \exp \left(k_{D} D_{i j}\right) \exp \left(k_{S} S_{i j}\right)\left(1-n_{i j}\right) \xi_{i j}
$$

with a normalisation $N$ that guarantees $p_{i j}=1 . n_{i j}$ is the occupation number of the target cell $(i, j)$, ie $n_{i j}=1$ for an occupied cell and $n_{i j}=0$ otherwise. Similarly, $\xi_{i j}$ is a hindrance factor, which is 0 if a cell cannot be reached, eg due to the presence of a wall or other obstacle, and 1 otherwise.

3. Each pedestrian randomly chooses a target cell based on the transition probabilities $p_{i j}$ determined by step 2 .

4. Whenever two or more pedestrians attempt to move to the same target cell, the movement of all involved

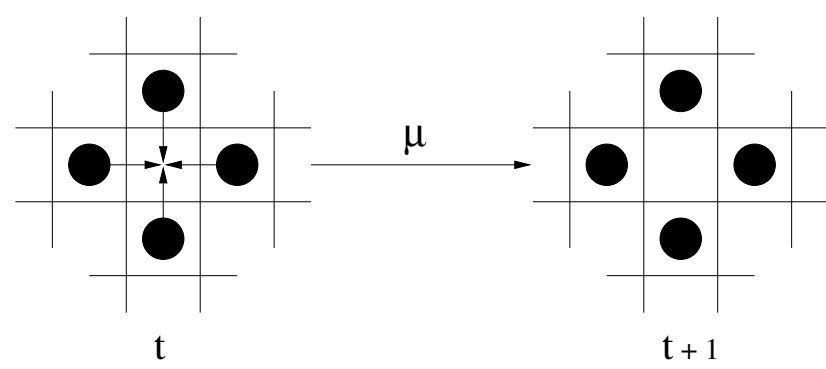

Figure 6 Refused movement due to the friction parameter $\mu$

particles is denied with probability $\mu \in[0,1]$ (see Figure 6), ie all pedestrians remain at their site (Kirchner, Nishinari et al 2003). This means that with probability $1-\mu$, one of the individuals moves to the desired cell. The one allowed to move is decided using a probabilistic method (Burstedde et al 2001; Kirchner, Nishinari et al 2003). In the following, $\mu$ is called 'friction parameter', since it has similar effects as friction between, for example, sand grains.

5. The pedestrians who are allowed to move perform their motion to the target cell chosen in step 3.D, at the origin cell $(i, j)$ of each moving particle, is increased by one: $D_{i j} \rightarrow D_{i j}+1$, ie $D$ can take any non-negative integer value. This corresponds to an ant dropping a pheromone. In contrast to the ant trail model, an arbitrary number of pheromones can exist at each site so that it is possible to model a varying strength of the trace.

The above rules are applied to all pedestrians at the same time (parallel update). The rules and their interpretation will be explained in more detail below.

In principle we can give each particle a preferred walking direction. From this direction, a $3 \times 3$ 'matrix of preferences' (Burstedde et al 2001) is constructed, which contains the probabilities for a move of the particle in a certain direction when interactions are neglected. The probabilities can be related to the average velocity and its longitudinal and transversal standard deviations (see Burstedde (2001) for details). So the matrix of preferences contains information about the preferred walking direction and speed. In principle, it can differ from cell to cell depending on the geometry and aim of the pedestrians.

\section{Interpretation of the rules}

To reproduce the collective phenomena discussed in a previous section, it is necessary to take into account the motion of all other pedestrians in a certain neighbourhood. This implies the introduction of non-local or longer-ranged interactions. In some continuous models this is done using 
the idea of a social force (Helbing and Molnar 1995; Helbing 2001). The approach described above is different. Since we want to keep the model as simple as possible we avoid using a long-range interaction explicitly. Instead, the concept of a 'floor field' was introduced that takes into account interactions between pedestrians and the geometry of the system (building) in a unified and simple way without loosing the advantages of local transition rules. The floor field modifies the transition probabilities in such a way that a motion into the direction of larger fields is preferred.

The 'dynamic floor field' $D$ is just the virtual trace left by the pedestrians. It is modified by the presence of pedestrians and has its own dynamics, ie diffusion and decay. Usually the dynamic floor field is used to model a ('longranged') attractive interaction between the particles. Each pedestrian leaves a 'trace', ie the floor field of occupied cells is increased. Diffusion and decay lead to a broadening, dilution and, finally, the vanishing of the trace.

The 'static floor field' $S$ does not evolve with time and is not changed by the presence of pedestrians. Such a field can be used to specify regions of space that are more attractive, eg emergency exits or shop windows.

Since the total transition probability is proportional to the dynamic floor field, it becomes more attractive to follow in the footsteps of other pedestrians. This effect competes with the preferred walking direction and the effects of the geometry encoded in $S_{i j}$. The relative influence of the contributions is controlled by the coupling parameters $k_{S}$ and $k_{D}$. These depend on the situation to be studied. Consider, for example, a situation where people want to leave a large room. Normal circumstances, where everybody is able to see the exit, can be modelled by solely using a static floor field, which decreases radially with the distance from the door. Since transitions in the direction of larger fields are more likely, this will automatically guarantee that everybody is walking in the direction of the door. If, however, the exit cannot be seen by everybody, eg in a smoke-filled room or in the case of failing lights, people will try to follow others, hoping that they know the location of the exit. In this case, the coupling to the dynamic floor field is much stronger, and the static field has a considerable influence only in the vicinity of the door.

The influence of the friction parameter $\mu \in[0,1]$ (see Kirchner, Nishinari et al (2003) for details) in step 4 of the update rules, which describes clogging effects between the pedestrians, will now be discussed. Due to the use of parallel dynamics, it might happen that in step 3 two (or more) pedestrians choose the same target cell. Such a situation is called a 'conflict'. Since the hard-core exclusion allows at most one particle per cell, at most one person can move. If friction is included, the movement of all involved particles is denied with the probability $\mu$, ie all pedestrians remain at their site (see Figure 6). This means that with probability $1-\mu$, one of the individuals moves to the desired cell. The particle that actually moves is then determined by a probabilistic method, eg by choosing one of them with equal probabilities.

The effects of this friction parameter are similar to those of contact friction in granular materials, which explains the name. It can be interpreted as a moment of hesitation when people meet and try to avoid each other. It is a local effect that can have enormous influence on macroscopic quantities like flow and evacuation time (Kirchner, Nishinari et al 2003). Note that the kind of friction introduced here only influences interacting particles, not the average velocity of a freely moving pedestrian.

\section{Results}

The model defined in the previous section is easy to implement on a computer. Due to its simplicity, very efficient Monte Carlo simulations, even of large crowds, are possible. In the following, some interesting results are highlighted.

\section{Collective effects}

The model is able to reproduce various fundamental phenomena described in the section Collective phenomena, such as lane formation in a corridor, herding and oscillations at a bottleneck (Burstedde et al 2001; Kirchner and Schadschneider 2002). This is an indispensable property for any reliable model of pedestrian dynamics, especially for discussing safety issues.

As an example, a simulation of counterflow in a corridor is shown in Figure 7. One can clearly see that in the lower part of the figure lanes have formed, whereas in the upper part the motion is still disordered.

In simulations of an evacuation process from a large room with only one exit, three different regimes can be observed (Kirchner and Schadschneider 2002) depending on the values of the coupling constants $k_{S}$ and $k_{D}$ to the static and dynamic field, respectively. In these simulations, the pedestrians did not have a preferred direction of motion. The only information about the location of the exit was 


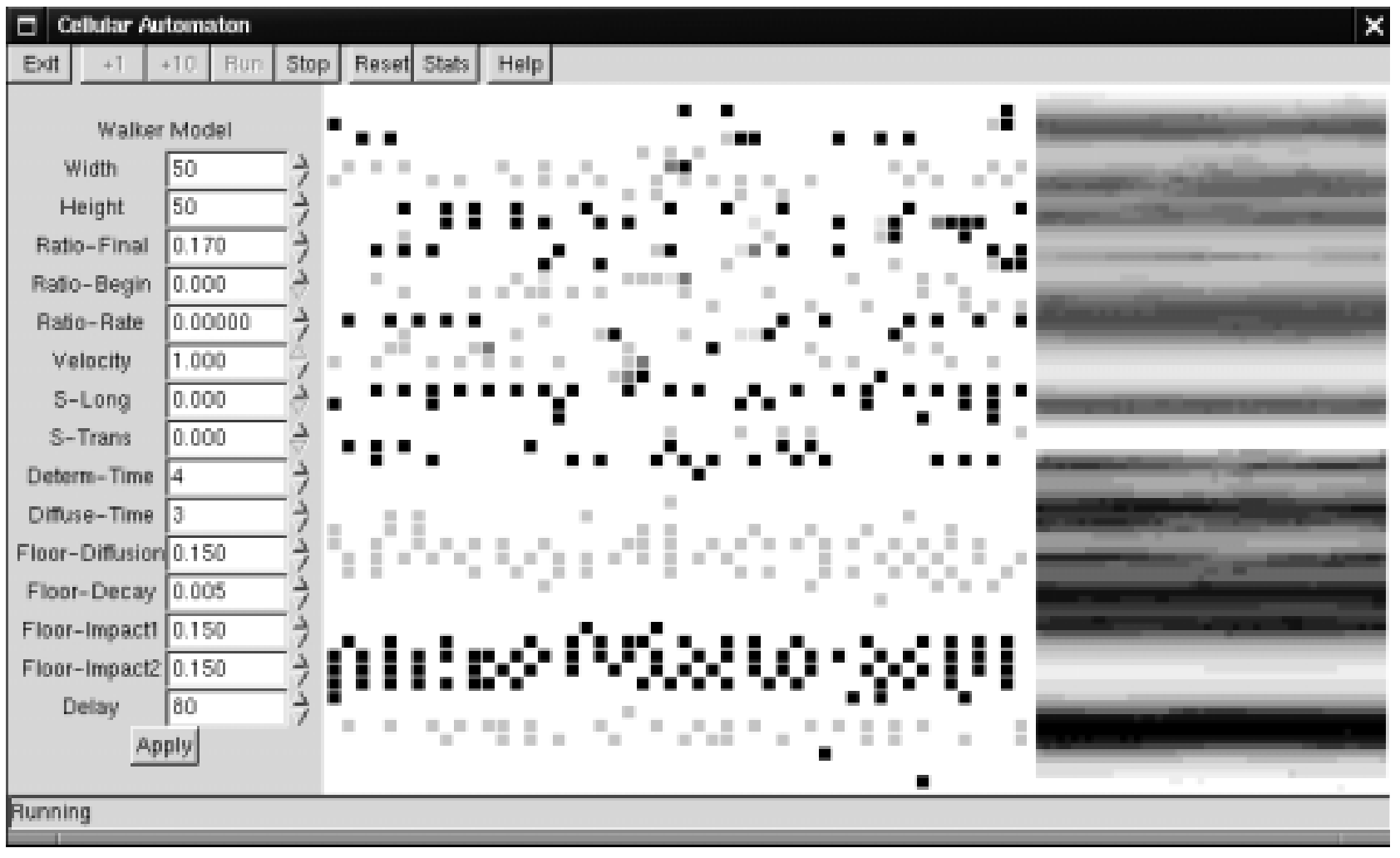

Figure 7 Snapshot from a simulation of a corridor with two groups of pedestrians moving in opposite directions. The left part shows the parameter control. The central window is the corridor and the light and dark squares are right- and left-moving pedestrians, respectively. The right part shows the floor fields for the two species.

obtained through the static floor field, which increases in the direction of the door.

For strong coupling $k_{S}$ and very small coupling $k_{D}$ we find an 'ordered regime'. This corresponds to a situation where all pedestrians have a good knowledge about the location of the exit and try to go there using the shortest path. At large densities, this leads to clogging at the door, since many pedestrians arrive there trying to leave the room. On the other hand, the 'disordered regime' is characterised by strong coupling $k_{D}$ and weak coupling $k_{S}$. This describes situations where the individuals do not know the exact location of the exit, eg in a smoke-filled room or in the case of failing lights. In such situations, people try to follow others, hoping that they know better where to go. This 'herding behaviour' is typical for 'panic situations'. Between these two regimes an 'optimal regime' exists where the combination of interaction with the static and the dynamic floor fields minimises the evacuation time (Kirchner and Schadschneider 2002).

In such evacuation simulations, counter-intuitive effects can be observed. Usually, one would expect that increasing the coupling $k_{S}$ to the static field would lead to a decrease of the evacuation time. For large $k_{s}$ people try to use the shortest way to the exit, whereas for small $k_{S}$ they perform a random motion, ie the effective velocity should increase with $k_{S}$. However, this is not always true.

Figure 8 shows the influence of the friction parameter on the evacuation time $T$. As expected, $T$ is monotonically

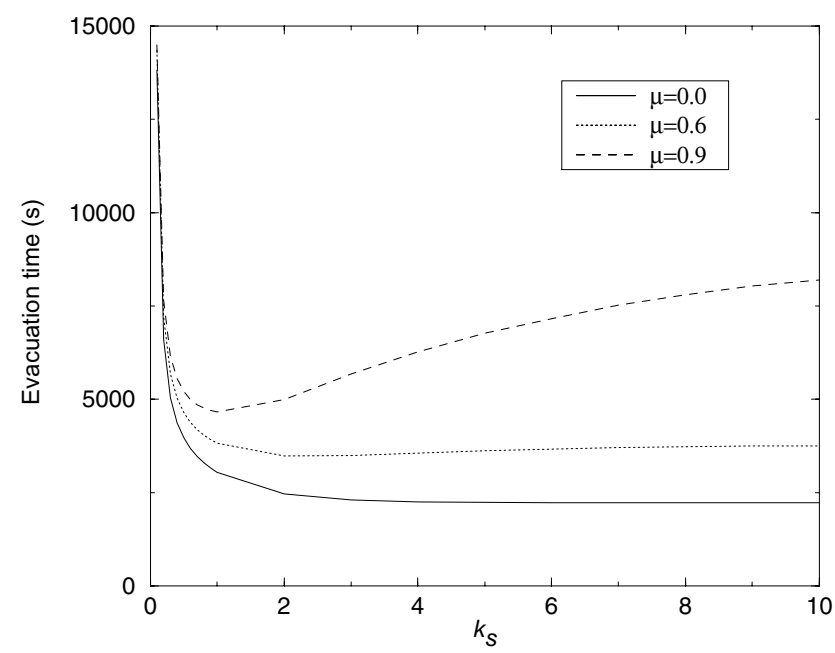

Figure 8 Dependence of evacuation times on the friction parameter $\mu$ as a function of $k_{s}$ for density $\rho=0.3$. 
increasing with $\mu$. For $\mu=0$ the evacuation time is monotonically decreasing with increasing $k_{S}$, as expected. For large $\mu$, however, $T\left(k_{S}\right)$ shows a minimum at an intermediate coupling strength $k_{S} \approx 1$. This behaviour is related to clogging at the door; here, the influence on the evacuation time. If $k_{S}$ is large, clogging is strong since all pedestrians arrive at the door quickly. For small and intermediate couplings, clogging is less strong because people arrive at very different times due to the almost random-walk-like behaviour. Therefore, fewer conflicts occur. Note that this observation is similar to the faster-isslower effect.

\section{An application: competitive versus cooperative behaviour}

An interesting experimental result (Muir et al 1996) shows that the motivation level has a significant influence on the egress time from a narrow body aircraft. The experiment was carried out with groups of 50-70 persons, where in one case (competitive), a bonus was paid for the first 30 persons reaching the exit, and in the non-competitive (cooperative) case, no bonus was paid. The time of the 30th person to reach the exit was measured ( $t_{\text {comp }}$ and $t_{\text {non-comp }}$, respectively) for variable exit widths $w$. The surprising result is $t_{\text {comp }}>t_{\text {non-comp }}$ for $w<w_{c}$, whereas $t_{\text {comp }}<t_{\text {non-comp }}$ for $w>w_{c}$. The critical width was determined experimentally to be about $70 \mathrm{~cm}$ (see Figure 9). This shows that competition is beneficial if the exit width exceeds a certain minimal value. For small exit widths, however, competition is harmful.

Within the framework of the model described above, competition is described as an increased assertiveness (large

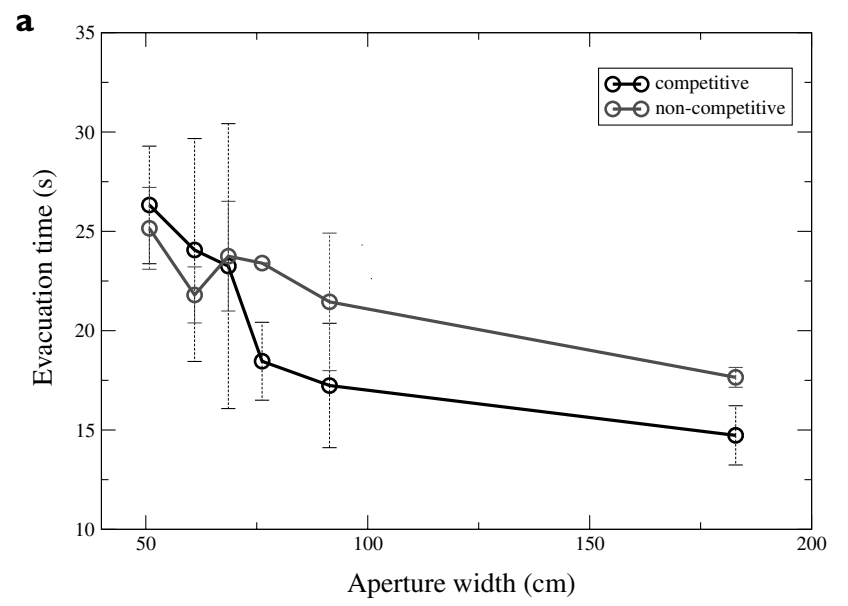

b

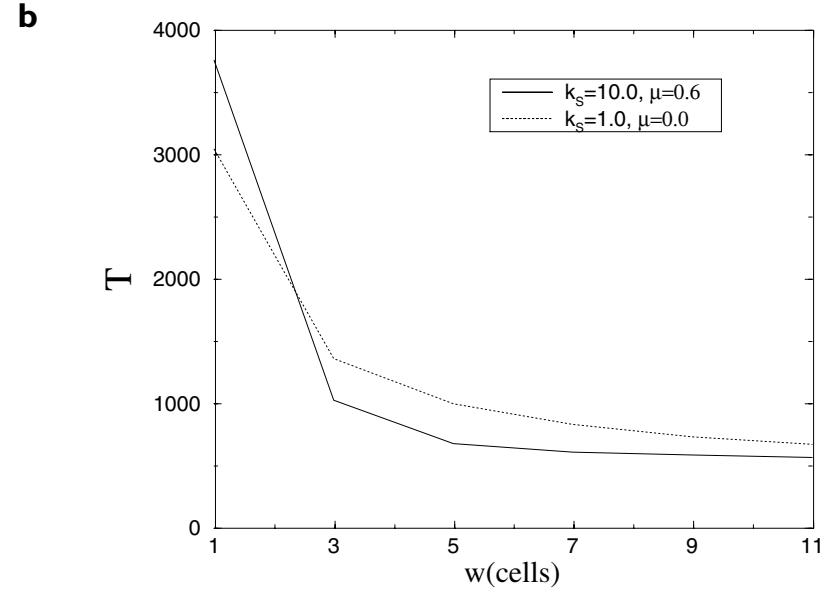

$k_{S}$ ) and at the same time strong hindrance in conflict situations, ie large $\mu$. Cooperation is then represented by small $k_{s}$ and vanishing $\mu$. This allows quantitatively distinguishing competition from cooperation and comparing the experimental results to simulations.

The experimental results have been reproduced qualitatively using a simplified scenario (Kirchner, Klüpfel et al 2003). Instead of a real aeroplane, an evacuation from a room without additional internal structure was simulated. Figure 9 shows typical average evacuation times for the noncompetitive and the competitive regime with an initial particle density of $\rho=0.03$ (116 particles). The door width is variable and ranges from 1 to 11 lattice sites. Clearly, the simulations are able to reproduce the observed crossing of the two curves at a small door width qualitatively. Without friction $(\mu=0)$, increasing $k_{S}$ alone always decreases $t_{\text {egress }}$. The effect is therefore only obtained by increasing both $k_{S}$ and $\mu$.

Thus, there are two factors that determine the egress of persons and the overall evacuation time in our scenario: walking speed (controlled by the parameter $k_{S}$ ) and friction (controlled by $\mu$ ). These parameters depend in a different way on the door width: the influence of the friction dominates for very narrow doors, which leads the crossing shown in Figure 9.

\section{Summary and conclusions}

We have introduced a stochastic cellular automaton to simulate pedestrian behaviour. $^{2}$ The general idea of our model is inspired by the phenomenon of chemotaxis, which is used by certain insects for communication. However,

Figure 9 (a) Experimental results for evacuation times for an aircraft with variable door width for competitive and non-competitive behaviour of the passengers. (b) Results from computer simulations of the cellular automaton model. 
pedestrians leave a virtual trace rather than a chemical one. This virtual trace has its own dynamics (diffusion and decay) that, for example, restricts the interaction range (in time). It is implemented as a dynamic floor field, which allows the use of local interactions only. Furthermore, we do not need to provide the pedestrian in the model with any 'intelligence'. Together with the static floor field, it offers the possibility to take different effects into account in a unified way, eg the social forces between the pedestrians or the geometry of the building.

The floor fields translate spatial long-ranged interactions into local interactions with memory. The latter can be implemented much more efficiently on a computer. Another advantage is an easier treatment of complex geometries, eg the formation of lanes in counterflow. Furthermore, counterintuitive results were found in evacuation simulations like the faster-is-slower effect.

The example presented here shows once more that nature has found efficient solutions to some difficult problems. In the case of pedestrian dynamics, the precise nature of interactions between people is difficult to determine. Therefore, it is not entirely clear whether our approach is just a technical trick or not. The virtual trace created by moving pedestrians might correspond to some representation of the path of others formed in the mind of each individual. Nevertheless, our results so far give a strong indication that there are more similarities between the motion of insects and 'intelligent individuals' than expected.

\section{Acknowledgements}

We thank D Chowdhury and H Klüpfel for helpful discussions and their collaboration on some of the results presented here.

\section{Notes}

1 In this paper, we use 'pedestrian' and 'particle' interchangeably.

2 Further information and $\mathrm{Java}^{\mathrm{TM}}$ applets for the scenarios studied here can be found at http://www.thp.uni-koeln.de/ as/

\section{References}

Ben-Jacob E. 1997. From snowflake formation to growth of bacterial colonies. II: cooperative formation of complex colonial patterns. Contemp Phys, 38:205-41.

Burstedde C, Klauck K, Schadschneider A et al. 2001. Simulation of pedestrian dynamics using a two-dimensional cellular automaton. Physica A, 295:507-25.

Camazine S, Deneubourg JL, Franks NR et al. 2001. Self-organization in biological systems. Princeton, NJ: Princeton Univ Pr.

Chowdhury D, Guttal V, Nishinari K et al. 2002. A cellular-automata model of flow in ant trails: non-monotonic variation of speed with density. $J$ Phys, A35:L573-7.

Chowdhury D, Santen L, Schadschneider A. 2000. Statistical physics of vehicular traffic and some related systems. Phys Rep, 329:199-329.

Helbing D. 2001. Traffic and related self-driven many-particle systems. Rev Mod Phys, 73:1067-141.

Helbing D, Farkas I, Vicsek T. 2000. Simulating dynamical features of escape panic. Nature, 407:487-90.

Helbing D, Molnar P. 1995. Social force model for pedestrian dynamics. Phys Rev E Stat Phys Plasmas Fluids Relat Interdiscip Topics, 51:4282-6.

Hölldobler B, Wilson EO. 1990. The ants. Cambridge, MA: Belknap.

Kirchner A, Klüpfel H, Nishinari K et al. 2003. Simulation of competitive egress behavior: comparison with aircraft evacuation data. Physica A, 324:689-97.

Kirchner A, Nishinari K, Schadschneider A. 2003. Friction effects and clogging in a cellular automaton model for pedestrian dynamics. Phys Rev E Stat Nonlin Soft Matter Phys, 67(5 Pt 2):056122.

Kirchner A, Schadschneider A. 2002. Simulation of evacuation processes using a bionics-inspired cellular automaton model for pedestrian dynamics. Physica A, 312:260-76.

Mikhailov AS, Calenbuhr V. 2002. From cells to societies. Models of complex coherent action. Berlin: Springer-Verlag.

Muir HC, Bottomley DM, Marrison C. 1996. Effects of motivation and cabin configuration on emergency aircraft evacuation behavior and rates of egress. Int $J$ Aviat Psychol, 6:57-77.

Nishinari K, Chowdhury D, Schadschneider A. 2003. Cluster formation and anomalous fundamental diagram in an ant-trail model. Phys Rev E Stat Nonlin Soft Matter Phys, 67(3 Pt 2):036120.

Schreckenberg M, Sharma SD, eds. 2001. Pedestrian and evacuation dynamics. Berlin: Springer-Verlag.

Wilson EO. 1971. The insect societies. Cambridge, MA: Belknap.

Wolfram S. 1986. Theory and applications of cellular automata. Singapore: World Scientific.

Wolfram S. 2002. A new kind of science. Illinois: Wolfram Media. 

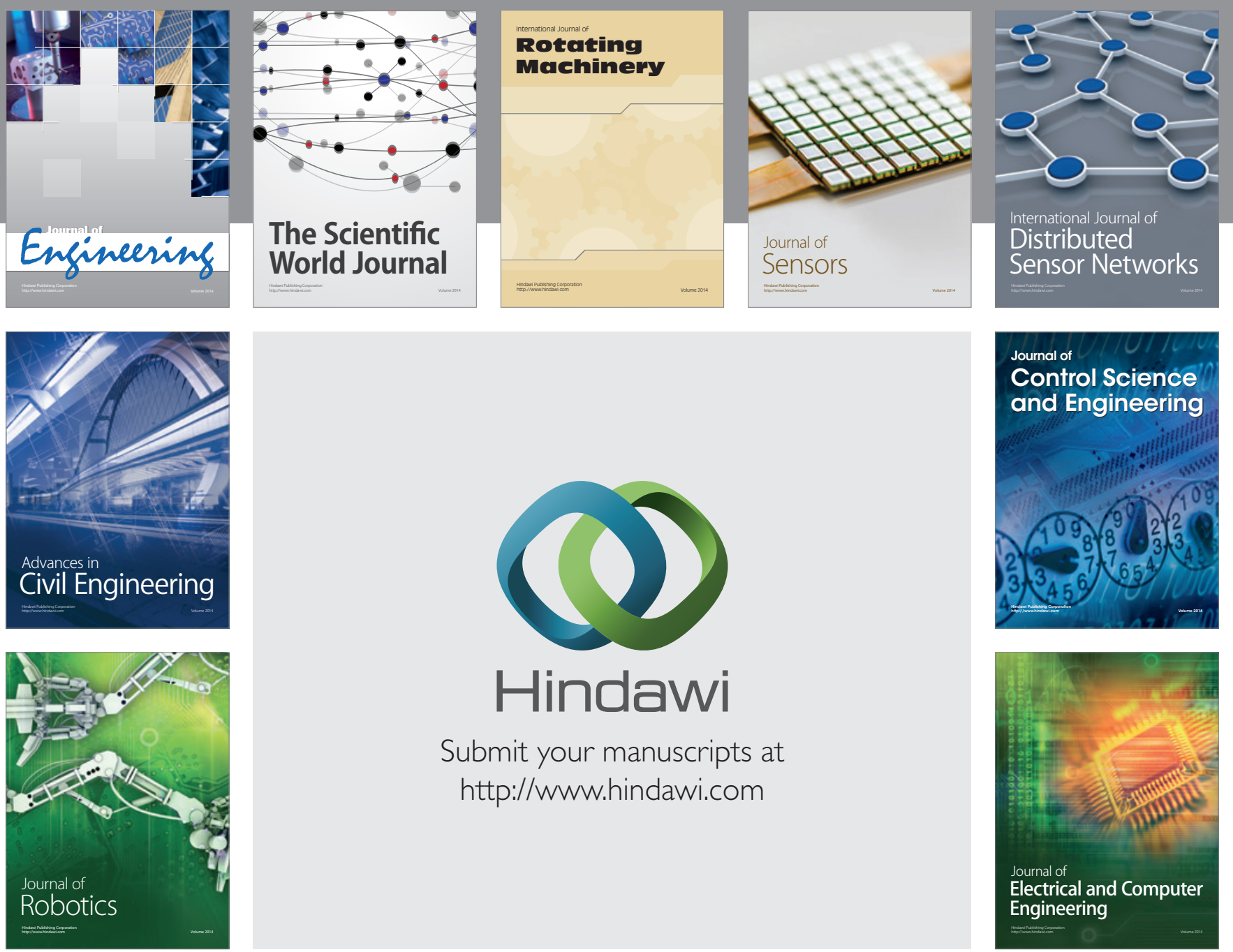

Submit your manuscripts at

http://www.hindawi.com
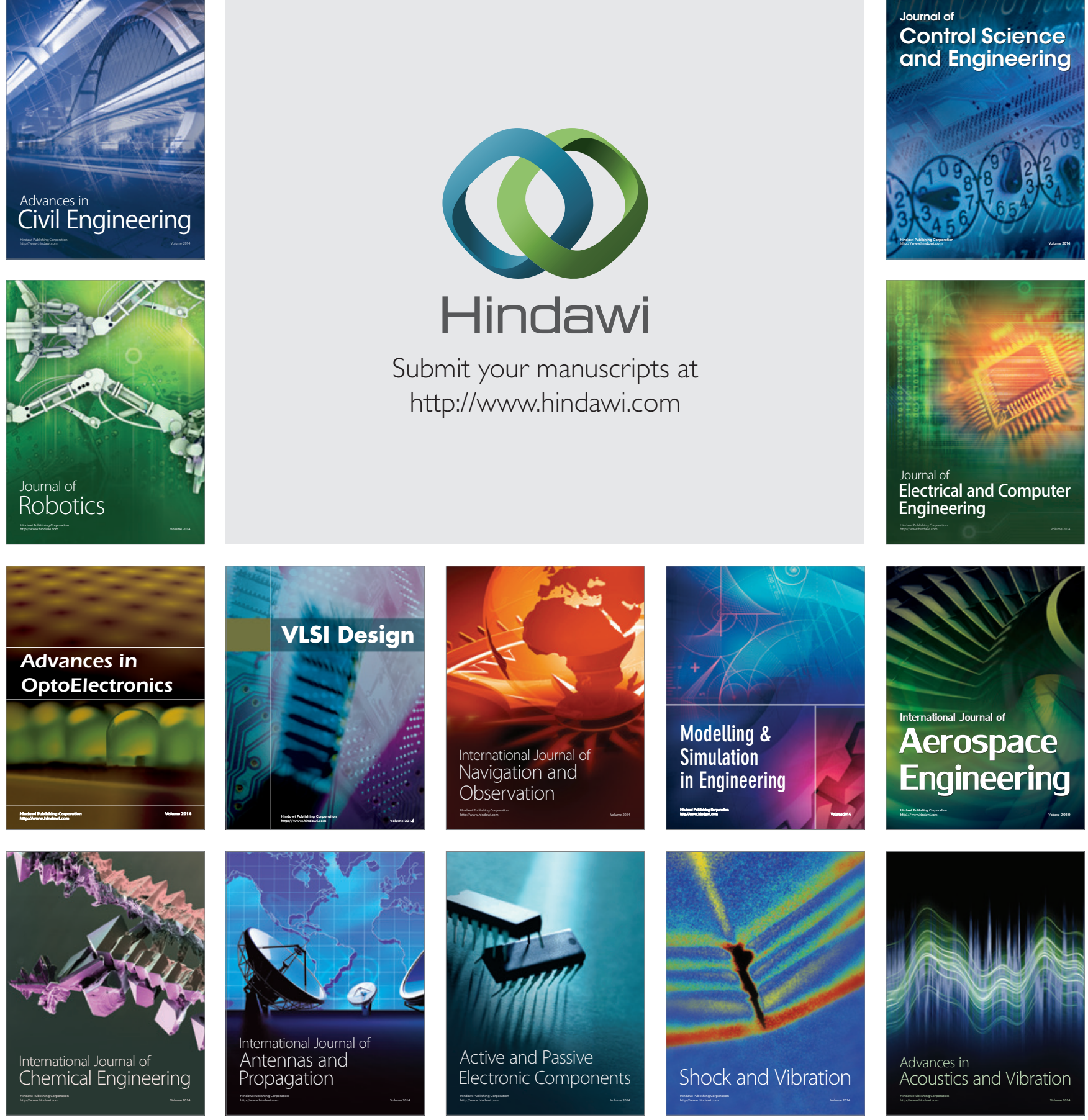